\title{
Linx
}

LinX Revue des linguistes de l'université Paris X Nanterre

59 | 2008

Les conjonctions en diachronie : parcours sémantiques

\section{La variation que / ce que et la formation des locutions conjonctives en français}

\section{Bernard Combettes}

\section{CpenEdition}

\section{Journals}

Édition électronique

URL : http://journals.openedition.org/linx/621

DOI : 10.4000/linx.621

ISSN : 2118-9692

Éditeur

Presses universitaires de Paris Nanterre

Édition imprimée

Date de publication : 1 décembre 2008

Pagination : 15-32

ISSN : 0246-8743

Référence électronique

Bernard Combettes, «La variation que / ce que et la formation des locutions conjonctives en français », Linx [En ligne], 59 | 2008, mis en ligne le 01 janvier 2012, consulté le 30 avril 2019. URL : http://journals.openedition.org/linx/621; DOI : 10.4000/linx.621 


\title{
La variation que / ce que et la formation des locutions conjonctives en français
}

\author{
Bernard Combettes \\ Nancy 2 \& UMR-ATILF
}

La formation des locutions conjonctives, problématique abordée dans cette étude, doit être replacée dans le cadre général de l'évolution syntaxique du français. L'une des tendances de fond de ce mouvement est constituée par le passage d'un système à déclinaison, caractéristique du latin classique, à un système qui, dépourvu de ce marquage morphologique des fonctions, exprime les diverses relations syntaxiques par des faits de position et par l'emploi des prépositions. Cette modification fondamentale, qui conduit d'une langue de type non configurationnel, à syntagmes faiblement structurés, vers une langue de type configurationnel, à syntagmes fortement hiérarchisés, va de pair, de façon assez naturelle, avec l'évolution, bien connue, qui mène d'un système synthétique à un système analytique, ce qui explique le développement des expressions complexes, des locutions, en particulier en ce qui concerne les familles des prépositions et des conjonctions. Si les locutions conjonctives ne sont pas absentes du latin (l'expression de sémantismes particuliers entraînant par exemple des formes comme simul atque : aussitôt que ou antequam : avant que), de la même façon que les conjonctions simples sont évidemment loin de disparaître en français, il n'en reste pas moins que se développent, dès l'ancien français, des séries de conjonctions complexes, dont certaines disparaissent au cours de l'histoire de la langue. S'opèrent ainsi divers processus de grammaticalisation, ces locutions étant formées à partir d'éléments qui, dans la plupart des cas, se combinent avec le subordonnant que ou, plus rarement, avec le relatif où. On peut considérer que pour les locutions les plus anciennes, l'étape de réanalyse est déjà bien engagée dès l'ancien français, la première 
partie de la locution n'étant plus interprétée avec sa valeur de départ, comme un adverbe ou comme une préposition ; en revanche, pour les expressions qui se créent durant le moyen français, on assiste au figement progressif des divers éléments qui les constituent et au changement de catégorie inhérent à la grammaticalisation, tant du point de vue syntaxique que du point de vue sémantique. Il convient de remarquer que cette réorganisation des marqueurs de subordination, mouvement dont les débuts ont été étudiés par Dardel (1983) et par Herman (1963) pour l'ensemble des langues romanes, concerne toutes les grandes sous-classes de propositions circonstancielles : hypothétiques (pourvu que, à condition que, au cas que / où,...), finales (à fin que, pour que,...), causales (pour ce que, puis que, ...), temporelles (lors que, dès que, après que, ...), concessives (bien que, combien que, ...). Dans la mesure où ces diverses catégories sémantiques témoignent, au-delà de quelques points communs qui les opposent aux subordonnées régies, de propriétés différentes, en particulier dans le domaine pragmatique et textuel et dans leur relation avec l'ensemble de l'énoncé, il serait sans doute indispensable d'analyser chacun des microsystèmes qu'elles constituent ; cette tâche a déjà été bien entreprise pour certaines de ces sous-catégories (Imbs, 1956 ; Soutet, 1992 ; Bertin, 1997 ; Verjans, 2009 ; Bat-Zeev Shyldkrot \& Kemmer, 1988, Combettes, 2006). Nous nous attacherons ici à un aspect particulier de cette évolution, aspect qui peut être considéré comme transversal, car il ne se trouve pas limité à une catégorie précise de subordonnée : il s'agit de l'alternance que / ce que, qui conduit à la formation d'une double série de locutions, les unes constituées d'un élément adverbial ou d'une préposition et de que (alors que, dès que ...), les autres comportant le démonstratif ce dans des séquences comme par ce que, avant ce que ... Nous sommes donc en présence, durant le moyen français et, pour certaines locutions, jusqu'au français préclassique, d'expressions qui offrent deux variantes (après que / après ce que, sans que / sans ce que, dès que / dès ce que ...), situation dont il faut essayer de déterminer le statut dans le système de la langue : variation libre ou opposition pertinente entre un tour marqué et un tour non marqué ? Cette alternance a déjà été commentée dans les travaux que nous avons mentionnés plus haut ; elle a été prise en compte, dans une diachronie large, jusqu'à l'aboutissement en français moderne, par Léard (1986) et par Pierrard (1993). Pour notre part, c'est essentiellement sur le moyen français que nous mettrons l'accent et que nous ferons porter nos observations; il nous semble en effet que c'est à cette période, et, plus particulièrement, au milieu du XVe siècle, que trouve son point d'achèvement le déséquilibre qui conduira à une répartition claire des formes en que et des formes en ce que, répartition caractéristique du français classique et du français moderne.

Si l'on excepte le cas des formes de participe, le premier élément de ces locutions relève essentiellement de la catégorie de l'adverbe ou de celle de la préposition. Il convient d'abord de considérer à part le cas des prépositions "pures », qui n'appartiennent qu'à cette seule catégorie, comme à, de, en, ..., et qui ne peuvent entrer dans les systèmes corrélatifs; introduisant obligatoirement un constituant à valeur nominale, elles apparaissent naturellement, pourrait-on dire, dans des séquences avec démonstratif, et des combinaisons telles que * à que, * de que, ou * en que, ne peuvent être attendues, dans la mesure où l'élément que n'est pas interprétable comme ce que, mais a obligatoirement la fonction d'un relateur. La présence du démonstratif semble donc 
indispensable. Si certaines formes complexes font difficulté, telles que dès que ou pour que, on doit toutefois remarquer que les variantes dès ce que et pour ce que sont attestées et que l'élimination de la séquence avec démonstratif peut s'expliquer par des phénomènes d'analogie, d'uniformisation, dans le mouvement général que nous allons essayer de décrire. Ne nous attachant donc pas à l'examen des prépositions pures, qui n'autorisent pas l'alternance que / ce que, nous noterons cependant au passage que si des locutions formées sur ces éléments ne semblent pas avoir subi de changement au cours de l'histoire de la langue, elles ont toutefois connu une évolution dans leur fonctionnement syntaxique. Les conjonctions complexes à ce que et en ce que peuvent en effet, en ancien français, introduire des subordonnées circonstancielles et ne se voient pas limitées au rôle d'introducteur de proposition régie. Ainsi, dans les passages suivants :

1) A ce que li uns l'autre encontre / Sagremors sa lance peçoie (Queste du Graal)

[Au moment que l'un rencontre l'autre, Sagremort brise sa lance]

2) Li rois revint en sa tente: et en ce qu'il fu revenuz, uns vellez vint devant lui (Mort Artu)

[Le roi revint dans sa tente : et quand il fut revenu, un valet vint au devant de lui]

3) elle [l'ost] ne peüst tenir ensemble, a ce que tant de gent li queroient mal (Villehardouin)

['armée ne put rester unie, comme tant de gens lui voulaient du mal]

ces locutions, qui ne sont pas sous la dépendance du prédicat principal, prennent-elles une valeur temporelle, valeur interprétée d'ailleurs, dans le dernier exemple, comme une relation de causalité. La séquence de ce que parait faire exception, mais la préposition de se prête mal, par son sémantisme, à l'expression de la temporalité ou à celle de la causalité, et on voit mal quelle serait l'interprétation d'une relation circonstancielle comme de ce que $\mathrm{P}_{1}, \mathrm{P}_{2}$, un tel enchaînement conduisant, nous semble-t-il, à un rapport de rection (de ce que $\mathrm{P}_{1}$, je déduis / conclus que ...). Une réorganisation s'est donc opérée, qui a conduit à une spécialisation syntaxique des formes en ce que, réservées à l'introduction des propositions dépendantes. Cette répartition - à laquelle par ce que semble faire exception - est à replacer dans l'évolution générale que nous avons rappelée au début de cet article : la perte du marquage assuré par la déclinaison s'accompagne d'une hiérarchisation plus forte des divers constituants de l'énoncé et de la spécialisation des catégories morphosyntaxiques. La réalisation de cette tendance dans le domaine de la subordination se traduit ainsi par une opposition de plus en plus nette entre propositions régies et propositions circonstancielles, cette distinction étant renforcée par la systématisation des formes à ce que, de ce que, en ce que devant les subordonnées « essentielles ». D'une manière inverse, en quelque sorte, les expressions en que se voient réservées aux subordonnées non régies, de statut syntaxique plus ou moins périphérique. Cette régularisation, fondée sur la fonction de la proposition introduite, constitue sans doute un des facteurs principaux de l'élimination des tours 
en ce que formés sur des adverbiaux (avant ce que, après ce que, ...). Il apparait toutefois que, dès la création des locutions, un déséquilibre dû à l'origine même de ces tours, peut être relevé entre que et ce que. La tendance à la distinction entre les types de marqueurs s'exerce ainsi sur un terrain déjà favorable au changement. Ce sont les raisons de ce déséquilibre que nous allons à présent observer. Dans la mesure où l'origine de ces locutions et l'histoire de leur formation permettent de justifier les diverses propriétés qui les caractérisent, il n'est pas inutile de rappeler rapidement comment le système du latin présente déjà les variations qui se trouveront attestées dès les origines du français. Sur ce point, il est intéressant de noter que les locutions françaises sont rarement le résultat de l'évolution d'une forme latine ; si on peut penser que avant que ou puis que sont des calques, des "imitations", de ante quam et de post quod, des expressions comme dès que / dès ce que, sans que / sans ce que sont bien des créations propres au français, qui se grammaticalisent durant l'ancien et le moyen français. Ce n'est donc pas tant l'étymologie de tel ou tel terme constituant les locutions qui est ici le point important que le maintien de schémas de construction, de «patrons » syntaxiques qu'offrait le système de la langue latine. Se continuent ainsi des régularités sémantiques et pragmatiques qu'il convient de prendre en compte dans l'étude du processus de grammaticalisation, la différence de statut morphosyntaxique du premier élément de la locution jouant alors un rôle primordial.

Dans son système de subordination, face à une famille de conjonctions simples (ut, cum, quod, ...), le latin offre la possibilité de structures corrélatives dans lesquelles le rapport d'hypotaxe se traduit par la mise en relation de deux termes, dont l'un est inséré dans la proposition enchâssante, alors que l'autre joue le rôle d'introducteur de la subordonnée. Ces constructions corrélatives se répartissent en deux sous-catégories, que l'on retrouvera en ancien français, fondées sur la nature et la fonction du premier élément de l'expression complexe. La première de ces catégories traduit d'ordinaire les divers aspects de la comparaison et les relations de conséquence ; d'une corrélation entre l'adverbe tam $(=$ autant $)$ et $u t$, fondée sur l'expression du degré :

4) quis tam demens est ut sua voluntate maereat?

(Cicéron, in Ernout \& Thomas, 344)

[quel est celui qui est assez fou pour qu'il puisse s'affliger de son plein gré ?]

on passe facilement à une relation de consécution, avec ita $(=$ ainsi $)$...ut:

5) scripta lex ita diligenter est ut (...) appareat (id.)

[la loi a été rédigée si scrupuleusement (...) qu'il apparaît que (...)]

Ce schéma général, qui comporte dans la plupart des cas un élément adverbial comme premier terme de la corrélation, s'étend à d'autres valeurs circonstancielles et, plus particulièrement, à l'expression de la temporalité. C'est ainsi qu'une forme comme priusquam (avant que) est formée à partir du comparatif prius (litt. plus avant), dont on retrouve l'exact équivalent dans les locutions françaises aussi tôt que et plutôt que ou, avec 
un premier élément qui n'est pas un comparatif au sens strict, mais un marqueur de série, l'ancien français premier que (avant que). Il faut également noter que l'origine de ces tours est soulignée, en quelque sorte, par la possibilité de la tmèse, aussi bien en latin :

6) hac lege ante omnia veneunt ... quam gleba una ematur (Cicéron)

[par cette loi tout est vendu avant qu'une seule motte de terre soit achetée]

qu'en ancien français :

7) Il fu avant un an et demi que il revenist au roi

(Joinville)

[ll se passa un an et demi avant que il revînt auprès du roi]

Le deuxième type de corrélation, qui apparait sous de nombreuses variantes dans la syntaxe latine, est constitué par la mise en rapport d'une forme anaphorique, d'ordinaire du paradigme des démonstratifs is ou bic, avec un subordonnant, quod ou $u t$ :

8) cum hoc proprium sit animantium ut aliquid appetent

(Cicéron )

[comme c'est le propre des êtres vivants d'avoir quelque appétence]

La tendance à généraliser quod comme seul marqueur de subordination a peu à peu éliminé une forme comme $u$, qui disparaît du système roman. Cette corrélation est sans doute facilitée par la nature pronominale de quod, et peut ainsi être rapprochée de la subordination relative, dans la mesure où le démonstratif remplit une fonction nominale dans la proposition matrice et joue ainsi le rôle d'un antécédent. Dans l'exemple que nous venons de citer, le pronom hoc a la fonction syntaxique de sujet; il peut également être utilisé à l'ablatif, avec une valeur circonstancielle de cause, ce qui conduit à la grammaticalisation d'une expression comme eo ...quod (pour cela ... à savoir que ...) ou, avec d'autres éléments comme ideo, propterea (pour cette raison) : ideo ... quod; propterea ... quod. La réunion des deux éléments de la locution conduit à des séquences figées (eo quod, ideo quod, propterea quod), la corrélation disparaissant au profit d'une expression complexe. On peut voir là un indice de l'évolution vers le type analytique, évolution qui devient encore plus nette, à l'époque tardive, lorsque se développent des tours prépositionnels comme ex eo quod (à partir de cela que ...) ou pro eo quo (pour cela que...), dans lesquels la préposition vient suppléer à un ablatif qui n'était plus senti comme tel (cf. Herman, 1963).

Nous allons à présent examiner rapidement comment ces deux grands types de construction des locutions conjonctives se retrouvent dès l'ancien français, avant d'analyser comment se réalise l'opposition des formes en que et des formes en ce que. Dans les tours corrélatifs qui mettent en relation un adverbial et que, le rapprochement avec les structures comparatives, nettement perceptible en latin, se justifie également en français. Considérons par exemple le cas du comparatif d'égalité, dont le deuxième élément est ordinairement introduit par com(e): 
9) li rois qui tant les amoit com s'il les eust de sa char engendrez ... (Queste du Graal)

[le roi, qui les aimait autant que s'il les avait engendrés de sa propre chair... ]

la réunion de l'adverbe tant et de com conduit à la création d'une locution dans laquelle la notion de quantité est appliquée au domaine de la temporalité, autant que évoluant vers : aussi longtemps que, d'où pendant que:

10) Et tant com li emperere cele parole at dite ... oït on vent venir (Pèlerinage de Charlemagne)

[Et pendant que l'empereur a dit cette parole, on entendit le vent venir]

Cette structure se retrouve d'ailleurs dans l'expression tandis que que l'on peut considérer comme issue du latin tamdiu, elle-même formée de l'intensif tam et de l'adverbial diu (longtemps)

11) les ymages que Lancelos avoit portretes tandis comme il demora leanz (Mort Artu)

[les images que Lancelot avait dessinées quand il séjourna là]

L'évolution générale des tours comparatifs présente, entre autres caractéristiques, le remplacement de comme par la forme non marquée que, qui s'étend à l'expression de l'égalité. Cette extension se manifeste également dans le cas de la locution conjonctive :

12) Tandis que nous mangiens, il vindrent ...

(Joinville)

[Tandis que nous mangions, ils vinrent]

Sur le modèle légué par le système latin se crée ainsi en ancien français une série de corrélations dont le premier constituant peut fonctionner seul comme adverbial mais va également entrer, par le phénomène de figement, dans une expression complexe introductrice de proposition dépendante; c'est par exemple, pour marquer la concomitance, le cas de formes comme dementres, issu de dum interim (pendant ce temps) et de ses nombreuses variantes (endementres, endementiers, dementiers, etc.), qui n'ont pas de valeur comparative, mais qui s'alignent sur la structure illustrée par tandis que:

13) Dementres que li fers est caus / Le doit on batre (Jean d'Arras)

[Pendant que le fer est chaud on doit le battre]

Le rapprochement de ces constructions avec les tours comparatifs peut se trouver justifié par la présence, dans certains énoncés, d'un marqueur comme tant, corrélé d'ailleurs à cum dans l'exemple suivant:

14) An tant dementres cum il iloec unt sis (...)

(La vie de saint Alexis) 
[en aussi pendant le temps comme $=$ pendant qu'ils sont assis là]

Il est intéressant de noter que l'adverbe ains, utilisé lorsqu'il s'agit d'exprimer l'antériorité, est à rattacher à un étymon antius, qui peut être considéré comme une formation de comparatif (plus avant = auparavant) ; ici encore, la corrélation avec que entraîne une relation identique à celle de la comparaison entre deux contenus propositionnels ; même si la réunion des deux éléments de la locution apparaît comme très ancienne et bien implantée (cf. Imbs, 1956), l'ancien français présente encore des cas de tmèse, bon témoignage de l'origine corrélative du tour, comme dans :

15) Mais ainz fu la novele dite au chastel que il i venissent (Yvain)

[Mais avant la nouvelle fut dite au château qu'ils y viennent]

16) Einz i morrat que cuardise facet

(Chanson de Roland)

[Avant (plutôt) il y mourra qu'il fasse couardise]

Comme dans le cas de la concomitance, ce type de structuration de la phrase complexe s'étend aux expressions qui ne relèvent pas du domaine de la comparaison, qu'il s'agisse de ainçois (ante eis = avant cela) :

17) J'auroie ainçois maint pas marché que fusse venu au marché (Roman de Renart)

[J'aurai avant marché beaucoup de pas que je fusse venu au marché]

ou de avant:

18) La mer seroit avant tarie qu'ele fust de cel mal garie

La mer serait tarie avant qu'elle fût guérie de ce mal

Ce qu'il convient de souligner, c'est, d'un point de vue sémantique, la relation qui s'établit entre les deux propositions. La structuration par corrélation semble mieux se prêter que les tours en ce que au changement d'ordre métaphorique qui conduit par exemple de la temporalité à des valeurs plus logiques; la présence du démonstratif permet de mettre davantage l'accent, comme nous allons le voir, sur le repère temporel, sur la relation chronologique qui s'établit entre les procès, alors que la corrélation, qui renvoie au contenu même des propositions, peut faire passer au second plan la valeur strictement temporelle. C'est le cas pour une locution comme plutôt que, qui est sortie du domaine temporel; c'est également ce que l'on peut remarquer avec einz... que et avant ... que dans les exemples (16) et (18), qui expriment l'irréalité du prédicat contenu dans la proposition introduite par que (il n'est pas possible qu'il fasse jamais couardise; il n'est pas possible qu'elle soit jamais guérie). Cette propriété est à considérer comme un facteur important dans l'établissement de la dissymétrie qui fera des tours en que les tours non marqués de l'opposition. La construction en ce que, si elle aboutit à la création de locutions qui, en surface du 
moins, paraissent avoir, une fois le figement réalisé, le même comportement syntaxique que les corrélations que nous venons d'évoquer, a toutefois une origine assez différente pour que les propriétés sémantiques et pragmatiques des deux familles de locutions ne se superposent pas totalement. On rappellera d'abord que la liaison d'un démonstratif, qui fonctionne comme antécédent, et d'une proposition en que est un phénomène général, qui dépasse le cadre des locutions conjonctives à valeur circonstancielle. La forme anaphorique peut en effet remplir la plupart des fonctions nominales, en particulier la fonction de sujet :

19) Certes, ce poise moi mout fort que je lui doie doner mort (Tristan) [Assurément, il m'est très pénible que je doive lui donner la mort]

ou celle de complément régi, en construction directe ou indirecte :

20) De ço se sont esmerveillié que il mort en tel maniere (Roman de Thèbes)

[ils se sont étonné de ce qu'il meure de telle manière]

Comme dans le cas des fonctions circonstancielles du démonstratif, les deux éléments de la corrélation peuvent se trouver réunis :

21) Mes ce que li cuens avoit tort li grieve forment et empire (Cligès)

[Mais le fait que le comte ait tort lui est très pénible]

Parallèlement, au plan sémantique, le démonstratif, dans la mesure où il joue le rôle d'un nominal, permet de donner une valeur référentielle au contenu de la proposition à laquelle il renvoie par cataphore. Ce sont là des différences importantes avec les corrélations de type comparatif et qui expliquent que les formes en ce que soient réservées à l'expression des rapports de temporalité. Tout se passe comme si la séquence ce que, dans les expressions avant ce que, dès ce que, etc. était interprétée comme (avant) le moment où, (dès) le moment où, la présence de ce empêchant, si l'on peut dire, une relation sémantique "directe» entre les deux propositions réunies en une structure complexe. On peut ainsi contraster les deux exemples suivants :

22) ne savez vos pas comment il m'est puis avenu que vos ne me veïstes? (Mort Artu)

[ne savez-vous pas ce qui m'est arrivé depuis que vous ne me vîtes ?]

23) après ce que le roy d'armes aura crié (...) alors vendra ledit roy d'armes ou milieu des lisses

[après que le roi d'armes aura crié, alors ledit roi d'armes viendra au milieu des lices]

Dans le premier énoncé, il apparaît clairement que la détermination temporelle ne s'exerce pas par rapport à un point de repère qui serait un moment particulier, un 
événement qui marquerait le point de départ d'une certaine durée ; la négation du prédicat (ne me veïstes) montre bien qu'il s'agit de mettre en parallèle deux situations, l'important étant moins la chronologie relative qu'une relation entre les contenus des propositions: le fait d'être absent durant un certain temps et le fait de connaitre certains événements. Il n'en va pas de même avec le second extrait, dans lequel la succession se trouve soulignée par alors, qui pourrait être rapproché des nombreux énoncés du type : Après ce, alors ..., l'emploi du démonstratif permettant à la préposition de conserver sa pleine valeur temporelle et de jouer son rôle de marqueur circonstanciel. Ces propriétés de base -étymologiques, pourrait-on dire- nous semblent devoir être prises en compte pour que l'on puisse déterminer comment évoluent les deux types de locutions, dans un jeu d'oppositions que nous allons examiner à présent.

C'est avec les locutions qui introduisent des subordonnées temporelles que la variation que / ce que est la mieux représentée et que les oppositions se laissent le plus clairement percevoir, sans doute en raison du domaine sémantique concerné, et, plus particulièrement, du jeu de la modalisation et des aspects, qui constitue ici un facteur déterminant. Si chaque marqueur a ses caractéristiques propres, dues à son sémantisme (l'expression de l'antériorité ne posant pas exactement les mêmes problèmes que celle de la concomitance ou celle de la postériorité, par exemple), le fonctionnement des locutions apparait toutefois comme relativement homogène sur deux points au moins. En ce qui concerne l'aspect tout d'abord, le démonstratif $c e$, qui permet de traiter les événements dénotés comme des référents particuliers, va souvent renvoyer à des événements uniques, spécifiques, alors que l'aspect itératif ou, plus généralement, la valeur gnomique, se verront exprimés dans des propositions introduites par que. Cette opposition apparaît bien, avec l'alternance dès que / dès ce que, dans les deux exemples suivants :

24) Sa premiere oeuvre, dès qu'il estoit levez, estoit de servir Dieu (C. de Pisan) [sa première activité, dès qu'il était levé, était de servir Dieu]

25) ... Dès ce que ledict duc le sceüt, il fist dire ... que ... (Commynes) [dès que le duc le sut, il fit dire ... que ...]

Dans ces types de contextes, tout se passe comme si la forme en ce que était réservée à l'expression de la simple temporalité, les séquences en que pouvant correspondre à un plus riche éventail de valeurs. De façon moins systématique, une opposition aspectuelle comme celle de la perfectivité et de l'imperfectivité est parfois corrélée, du moins pour certaines locutions, à la variation qui nous intéresse ici ; dans l'extrait suivant, ce que est associé à l'accompli (être nê), tandis que la forme dès que va de pair avec un procès non accompli (apprendre).

26) On nourrit lez jeunes seigneurs ez delices ... Des ce qu'ilz sont naiz, c'est a dire dez qu'ilz apprennent a parler, ilz sont a l'escole de gouliardise ... (A. Chartier) 
[on élève les jeunes seigneurs dans les délices ... Dès qu'ils sont nés, c'est-à-dire dès qu'ils apprennent à parler, ils sont à l'école de la débauche ...]

C'est surtout dans le domaine de la modalisation du procès que va jouer pleinement l'alternance des deux types de formation. De façon relativement attendue, la présence du démonstratif dans la locution correspondra à des procès renvoyant à des états de choses présentés comme réalisés ou dont la réalisation est certaine. La forme en que, quant à elle, est utilisée lorsque survient une modalisation qui renvoie le procès au domaine du non réalisé (le cas le plus caractéristique étant celui de la négation), au domaine du possible, de l'incertain. Cette opposition est en quelque sorte une conséquence de la valeur et du rôle du démonstratif, qui focalise sur le moment de l'événement est sur la relation chronologique avec le prédicat principal. Avec que, c'est sur le procès que s'exerce la saillance, ou, plus exactement, sur la relation, tout autant logique que chronologique, qui unit les deux propositions. Dans les passages suivants, qui présentent les locutions construites sur avant, s'opposent la réalité du fait (on étuve et on saigne effectivement les lamproies, et c'est la chronologie des opérations qui est exprimée ici) et sa non réalisation (Hannibal n'a pas été averti de l'attaque d'Hostilius) :

27) aucuns seignent la lamproye avant ce que ilz les estauvent, et aucuns les estauvent avant ce qu'ilz les seignent ne eschaudent

(Le Ménagier de Paris)

[certains saignent la lamproie avant de l'étuver, et certains les étuvent avant qu'ils les saignent ou les échaudent]

28) Hostilius, avant que Hanibal fust advisé, luy courust sus hastivement (A. de La Sale)

[Hostilius, avant qu'Hannibal fût informé, l'attaqua rapidement ]

On peut percevoir, dans ce dernier exemple, une valeur sémantique qui vient s'ajouter à la notion d'antériorité, valeur qui serait soulignée par une expression comme avant même que; le fait que l'événement ne se soit pas produit conduit à inférer que le premier procès joue un rôle dans ce non accomplissement. Il n'en va évidemment pas de même lorsque, avec ce que, les faits rapportés sont tous deux du domaine du réel. Un effet comparable accompagne souvent l'emploi de dès (ce) que ou de depuis (ce) que. Si le sémantisme de dès est le même dans les deux extraits suivants, la relation de causalité, de conséquence nécessaire, se fait mieux sentir dans le deuxième exemple, que l'on pourrait paraphraser par : il suffit que ... pour que ... Il faut également noter que s'ajoute à cette valeur d'ordre logique celle de vérité générale que nous avons évoquée plus haut:

29) Dès ce que le roy Edouard eut gaigné ceste bataille, il tira au devant dudict prince de Galles

(Commynes) 
[dès que le roi Edouard eut gagné cette bataille, il alla au devant dudit prince de Galles]

30) des qu'elle (= la jalousie) entre dedens le cuer (...) tout bien s'enfuit (A. Chartier)

[dès que la jalousie entre dans le cœur (...) tout bien s'enfuit]

Le même type d'alternance survient avec depuis (ce) que, qui peut marquer une simple précision temporelle. C'est alors la forme avec démonstratif qui s'impose, avec sa valeur de le moment où :

31) depuis ce qu'ilz estoient couchiez, il avoit fait relever sa femme (Le Ménagier de Paris)

[depuis qu'ils étaient couchés, il avait fait relever sa femme]

Mais l'accent peut être mis sur les effets produits par la survenue ou par la durée du procès et l'on retrouve alors les mêmes relations qu'avec dès que, ou puis que par exemple :

32) Mais depuis que vertu et union fut chassée et deboutée d'icelluy royaulme, tout est tourné en fureur et debat

(La Marche)

[mais depuis que la vertu et l'union furent chassées de ce royaume, tout est devenu fureur et dispute]

Les énoncés contenant les deux variantes d'une même locution sont rares ; dans le passage suivant, extrait des Chroniques de Froissart, la première subordonnée temporelle, introduite par avant ce que joue un rôle de constituant cadratif et donne un repère chronologique pour la suite des événements rapportés. Aucune relation de causalité ne peut être établie entre cette indication et la proposition qui la suit et sur laquelle s'exerce sa portée (une grande tempête se leva). La deuxième subordonnée, introduite par avant que, ne met pas en avant le moment où les voyageurs se retrouvent, mais établit un rapport entre les contenus des deux propositions : s'ils n'avaient pas été obligés par la tempête à errer sur la mer, ils auraient pu se rassembler plus tôt. A l'inverse, en quelque sorte, de l'effet produit par dès que ou par depuis que, c'est une relation de conséquence contrariée qui est ici exprimée par la forme en que, dont le rôle se rapproche davantage de celui d'un connecteur que de celui d'un marqueur temporel :

33) un petit avant ce que mesires Robers d'Artois et la contesse de Montfort se departesissent dou havene de Plumude, uns grans tourmens se mist sus mer, (...) et furent plus de quinze jours waucrant sus la mer (...) avant que il se puissent tout remetre ensamble

(Froissart)

[peu avant que monseigneur ... partent du port de Plumude, une grande tempête se leva sur la mer ... et ils furent plus de quinze jours errant sur la mer avant qu'ils puissent se retrouver] 
On notera par ailleurs que les deux subordonnées remplissent une fonction différente au niveau informationnel. Comme l'implique son rôle de cadratif, la première temporelle appartient à la partie thématique de l'énoncé, ce qui va de pair, du point de vue syntaxique, avec son statut de constituant périphérique. La locution avant que, quant à elle, introduit une subordonnée nettement rhématique et sa place à la suite du syntagme verbal principal est quasiment obligatoire, du moins dans un texte en prose. Cette liaison étroite entre les contenus des propositions doit être rapprochée des cas de tmèse observables en ancien français. La corrélation qui s'établit, dans l'exemple suivant, entre ainçois (avant) et que entraîne le même effet de sens - la durée de la marche empêche l'arrivée rapide au marché, événement dont on ne sait d'ailleurs pas s'il est considéré comme réalisable ou non - que l'emploi de avant que dans le passage de Froissart que nous venons de citer :

34) J'auroie ainçois maint pas marché que fusse venu au marché (Roman de Renart)

[J'aurai avant marché beaucoup de pas que je fusse venu au marché]

La formation d'une expression complexe, avec réunion des deux éléments de la corrélation, n'a donc pas neutralisé les valeurs logiques qui étaient rattachées à l'emploi de que. C'est dans ce maintien que réside un des facteurs du déséquilibre qui fait de la forme en que le cas non marqué du système d'opposition. On constate en effet que les propositions introduites par cette dernière entrent dans les mêmes contextes que celles qui sont introduites par ce que et peuvent donc renvoyer également à des valeurs strictement chronologiques. Ainsi, lorsqu'il s'agit par exemple de marquer la simple antériorité, sans addition d'une valeur logique, les formes que et ce que semblent-elles être en variation libre. Dans l'exemple suivant, la subordonnée temporelle est introduite par avant que et n'entretient pas de valeur logique particulière avec le prédicat principal :

35) On lit que Pilate, avant que il fust envoyé a Rome de par l'empereur, il s'estoit meslé de finances

(Juvénal des Ursins)

[on lit que Pilate, avant qu'il soit envoyé à Rome par l'empereur, s'était occupé de finances]

Si l'on rapproche cet utilisation de avant que de l'emploi de avant ce que en (27), les deux variantes apparaissent bien comme des variantes libres dans ce type de contexte purement temporel. La même généralisation de que peut être constatée avec la locution dès que:

36) Dès que vous partirez de la porte, faictes que (...) vous n'arrestez point en chemin

(Jean de Bueil) 
[dès que vous vous éloignerez de la porte, faites en sorte de ne pas vous arrêter en chemin]

énoncé où la subordination ne correspond pas à une relation de causalité entre les propositions, et où dès que présente une valeur temporelle identique à celle de dès ce que dans l'exemple (29).

Deux raisons principales, qui se renforcent sans doute l'une l'autre, semblent ainsi permettre d'expliquer la répartition à laquelle a abouti l'évolution : la nature non marquée de que, qui introduit la subordonnée dans tous les contextes sémantiques, alors que ce que ne se combine qu'avec certains prédicats, la spécialisation de ce que comme introducteur de proposition régie, qui entraîne la généralisation des formes en que pour les subordonnées circonstancielles. La première de ces raisons est le résultat de l'origine même des tours, du type de construction qui a donné naissance aux locutions; la deuxième est une conséquence du mouvement de spécialisation des diverses catégories morphosyntaxiques qui caractérise l'histoire du français.

Nous terminerons cette étude par quelques remarques concernant la chronologie du changement. Comme nous l'avons déjà noté, le moyen français apparait, sur ce point comme sur bien d'autres faits de langue, comme une époque charnière. Si l'on essaye de déterminer avec le plus de précision possible - dans la mesure où les corpus le permettent - les étapes de l'évolution, on observe que c'est durant la première moitié du XVème siècle que s'opère une rupture assez nette. En prenant par exemple en compte quelques locutions temporelles attestées dans la base textuelle du DMF (cf. tableau), on constate toutefois une certaine dissymétrie entre les divers marqueurs, différences qui s'expliquent, nous semble-t-il, par le jeu de facteurs d'ordre sémantique, mais également par l'ancienneté plus ou moins grande des locutions. Ainsi pourra-t-on remarquer que les expressions construites sur des formes participiales (durant (ce) que; pendant (ce) que) ne présentent que très rarement la séquence avec démonstratif et que durant que et pendant que sont de loin les variantes les plus utilisées. La raison de ce déséquilibre réside probablement dans le fait qu'il y a là des créations relativement récentes, par rapport à des locutions comme après que ou avant que, par exemple. On notera que cependant, dont la formation date également du XIII ${ }^{\mathrm{e}}$ siècle, n'apparaît en locution conjonctive que sous la forme cependant que, le démonstratif étant encore interprété comme tel, ce qui empêche la création, par analogie de * cependant ce que. Parmi les locutions formées sur des adverbes, on regroupera avant, devant et depuis, que l'on opposer à après. Alors que les expressions avant que, devant que, depuis que, sont nettement majoritaires, face aux expressions en ce que dès le début du XVème siècle, la locution après ce que l'emporte sur après que dans la plupart des textes jusqu'en 1450 et ce n'est qu'à partir du milieu du siècle que la séquence après que commence à s'imposer. Du point de vue méthodologique, il serait évidemment indispensable de prendre en compte l'ensemble de l'histoire de ces tours, dès l'ancien français, pour tenter de déterminer si une telle dissymétrie est attestée dès l'origine ou si elle se constitue peu à peu. L'observation du moyen français permet néanmoins de constater que le mouvement de grammaticalisation est moins rapide dans le cas de après que, alors qu'il est presque réalisé pour l'autre famille d'expressions. Deux raisons au moins, dans l'optique que nous avons adoptée ici, peuvent être invoquées pour expliquer la place 
particulière occupée par après (ce) que. Il faut d'abord relever le fait que cette dernière expression se voit limitée au domaine de la temporalité, alors que les autres locutions peuvent prendre, dans certains contextes particuliers, que nous avons rapidement évoqués plus haut, des valeurs «logiques » dérivées de la temporalité, comme par exemple la conséquence nécessaire ou la consécution. L'alternance après que / après ce que se réalise bien comme l'opposition d'un tour marqué et d'un tour non marqué, l'un des traits pertinents importants étant constitué par la réalisation ou la non réalisation du procès, mais l'absence de valeurs métaphoriquement obtenues à partir de l'expression de la postériorité permet aux tours en ce de se maintenir relativement longtemps, l'expression de la chronologie " pure », soulignée par l'emploi du démonstratif résistant mieux que dans les autres expressions. Une deuxième particularité de après vient renforcer ce sémantisme de après ce que; il s'agit de la fréquence du syntagme prépositionnel après ce, utilisé en début d'énoncé avec une double valeur de circonstanciel cadratif et de lien anaphorique avec le contexte de gauche :

37) et lui fist la dame toute l'onneur qu'elle pot et lui compta l'usage et la maniere du païs. Et aprés ce, Aigres s'en ala couchier et reposer jusques au lendemain matin.

(Berinus)

Les termes comme avant, devant ou depuis se prêtent évidemment moins bien à une telle exploitation discursive. Leur sémantisme les conduit davantage à jouer un rôle informatif dans la partie rhématique de la phrase et à se trouver ainsi moins systématiquement associés à une forme comme ce. Le cas de dès que est un peu moins clair. Si l'on ne prenait pas en compte les occurrences fournies par le texte de Commynes, il serait possible de rattacher cette locution au sous-système constitué par avant, devant et depuis, ce qui n'aurait rien de surprenant, dans la mesure où dès que peut traduire la relation de conséquence nécessaire dérivée de la temporalité. Il reste toutefois que les Mémoires de Commynes offrent 48 exemples de dès ce que pour 29 de dès que, situation d'autant plus inattendue que l'usage de ce même auteur en ce qui concerne les autres locutions apparaît comme parfaitement conforme à l'évolution que nous venons de décrire : ce que n'est attesté que quatre fois, dans la séquence après ce que, dont on sait qu'elle correspond à un certain retard dans le mouvement de grammaticalisation. Devant de tels faits qui paraissent contredire les tendances générales du changement, on doit rappeler la nécessité de prendre en compte la typologie des textes et des discours. Cette spécificité de certaines parties du corpus se manifeste également avec les Registres du Châtelet, dans lesquels les formes en ce que sont bien mieux représentées que dans les autres textes, ce qui, dans ce cas particulier, peut constituer la trace d'une écriture archaïsante caractéristique d'un genre relativement figé. Si ces facteurs de type discursif ne doivent pas être négligés pour l'établissement d'une chronologie fine de l'évolution, il nous semble toutefois que ce critère n'intervient que marginalement et ne remet nullement en cause l'importance du milieu du XVème siècle dans ce qui constitue un des aspects de la réorganisation du système des catégories grammaticales. 


\begin{tabular}{|c|c|c|c|c|c|c|c|c|c|c|c|c|c|c|}
\hline & & \multicolumn{2}{|c|}{ après } & \multicolumn{2}{|c|}{ avant } & \multicolumn{2}{|c|}{ devant } & \multicolumn{2}{|c|}{ dès } & \multicolumn{2}{|c|}{ depuis } & \multicolumn{2}{|c|}{ durant } & \multirow{2}{*}{$\begin{array}{c}\text { pendan } \\
\text { que }\end{array}$} \\
\hline & & ce & que & ce & que & ce & que & ce & que & ce & que & ce & que & \\
\hline 1340 & Machaut & & & & 2 & & 1 & & 29 & & 2 & & 1 & 2 \\
\hline 1350 & Berinus & 19 & 4 & & 20 & & 2 & & & & 7 & & 7 & \\
\hline 1360 & Daudin & 2 & 2 & & 3 & & & & & & 1 & & 1 & \\
\hline 1370 & Oresme & 23 & 5 & & 24 & 1 & & 6 & 1 & & 1 & & 2 & \\
\hline 1370 & Miracles & 5 & 8 & 3 & 55 & 2 & 1 & & 6 & 1 & 19 & & 23 & \\
\hline 1385 & Deschamps & & 2 & & & & & & 2 & 1 & & & 4 & \\
\hline 1387 & Phebus & 3 & & & 21 & & 6 & & 13 & & 5 & & 5 & \\
\hline 1389 & Châtelet & 311 & 18 & 38 & 24 & & 1 & & & 21 & 19 & 3 & 19 & $c e: 19$ \\
\hline 1390 & $X V$ Joyes & 2 & 1 & & 3 & & & & & & 2 & & 2 & \\
\hline 1392 & J. Arras & 3 & & 4 & 27 & & 5 & 2 & & 1 & 11 & & 11 & \\
\hline 1394 & Ménagier & 17 & 3 & 3 & 25 & & & & & 1 & 6 & 1 & 6 & \\
\hline 1400 & Froissart & 4 & 1 & 5 & 57 & & 1 & & & & 21 & & 22 & \\
\hline 1402 & Gerson & 8 & & & 2 & & & & & & 3 & & 3 & \\
\hline 1404 & Pisan & 24 & 14 & & 19 & 1 & & & 2 & & 2 & & 2 & \\
\hline 1411 & Baye & 28 & & 7 & 3 & 1 & & & 1 & & 1 & & 1 & \\
\hline 1416 & Chartier & 5 & 2 & & 1 & 1 & & 2 & 5 & 1 & 2 & & 2 & \\
\hline 1421 & Fauquemb. & 34 & 1 & 14 & 1 & & & & & & 8 & 1 & 8 & 1 \\
\hline 1432 & Régnier & 1 & 2 & & 2 & & & & & & 1 & & 1 & \\
\hline 1435 & Juvénal & 28 & 3 & 1 & 45 & & & & & & 6 & 1 & 7 & \\
\hline 1450 & Crapillet & 5 & & & 10 & & 3 & & & & 2 & & 3 & \\
\hline 1456 & La Sale & 14 & 9 & & 25 & & 1 & & & & 2 & & 2 & \\
\hline 1456 & C. Nouv. & 2 & 26 & & 37 & & 2 & & 1 & & 2 & & 2 & \\
\hline 1460 & Roye & 15 & 46 & & 12 & & & & & & 4 & 3 & 8 & 2 \\
\hline 1461 & Bueil & 2 & 33 & & 39 & & 3 & 2 & 2 & & 7 & & 9 & 6 \\
\hline 1461 & Meschinot & & 1 & & 2 & & 3 & 2 & & & 1 & & 1 & \\
\hline 1465 & Bagnyon & 4 & 68 & & 37 & & 10 & & & & 4 & & 4 & \\
\hline
\end{tabular}




\begin{tabular}{|l|l|l|l|l|l|l|l|l|l|l|l|l|l|l|}
\hline 1466 & Ev. Quen. & & 10 & & 8 & & 3 & & & & 1 & & 1 & \\
\hline 1470 & La Marche & 2 & 17 & & 25 & & 1 & & 2 & & 7 & & 7 & \\
\hline 1472 & Fillastre & & 10 & & 3 & & & & 2 & & & & & \\
\hline 1489 & Commynes & 4 & 32 & & 68 & & 4 & 48 & 29 & & 18 & & 20 & 6 \\
\hline 1494 & de Phares & 4 & 30 & & 6 & & 3 & 2 & 1 & & 1 & & 1 & \\
\hline 1495 & La Vigne & & 18 & & 13 & & 9 & & 1 & & 3 & & 3 & 11 \\
\hline 1502 & Le Clerc & 4 & 27 & & 11 & & 3 & 1 & 2 & & 6 & & 6 & 1 \\
\hline
\end{tabular}

\section{RÉFÉRENCES BIBLIOGRAPHIQUES}

BAt-Zeev Shyldkrot, H. \& Kemmer, S., 1988, «Le développement sémantique des conjonctions en français : quelques concepts généraux », Revue Romane, 23, p. $9-20$.

BERTIN, A., 1997, L'expression de la cause en ancien français, Genève, Droz.

BURIDANT, C., 2000, Grammaire nowvelle de l'ancien français, Paris, SEDES.

COMBETTES, B., 1999, "Quelques caractéristiques de l'évolution du syntagme verbal en français ", Verbum, 21, p.271-287.

COMBETTES, B., 2006, «La formation des locutions conjonctives temporelles : le cas de dès que », in E. Moline \& al. eds, Les connecteurs temporels du français, Amsterdam, Rodopi, p. 1-19.

DARDEL de, R., 1983, Esquisse structurale des subordonnants conjonctionnels en roman commun, Genève, Droz.

DIK, S., 1997, The Theory of Functional Grammar, (2 vol.) Berlin, Mouton.

ERnOUT, A. \& ThOMAS F., 1969, Syntaxe latine, Paris, Klincksieck.

HERMAN, J., 1963, La formation du système roman des conjonctions de subordination, Berlin, Akademie-Verlag.

IMBS, P., 1956, Les propositions temporelles en ancien français, Publications de la Faculté des Lettres de l'Université de Strasbourg.

LÉARD, J.-M., 1986, «Le statut de ce que dans le système morphologique du français : une solution unitaire appuyée sur la diachronie », Modèles Linguistiques, VIII, 2, p. $7-45$.

LEHMAnN, C., 1988, «Towards a typology of clause linkage », in J. Haiman \& Thompson S. A. (eds), Clause combining in grammar and discourse, Amsterdam, Benjamins, p. 181-225. 
MarCHello-Nizia, C., 1995, L'évolution du français, Paris, Armand Colin.

PIERRARD, M., 1993, «A propos des hypothèses concernant la valeur de ce dans les subordonnées introduites par ce que », Actes du XX eongrès de Linguistique romane, Tome I : p. 591-602.

PIERRARD, M., 1995, «Système et histoire du système: l'évolution de ce que introducteur de subordonnées », Langue Française, 107, p. 111-124.

SOUTET, O., 1992, Etudes d'ancien et de moyen français, Paris, PUF.

VAn Valin, R. \& R. La POLLA, 1997, Syntax meaning and function, Cambridge University Press.

VERJANS, T., 2009, Essai de systématique diachronique: Genèse des conjonctions dans l'histoire du français (IX ${ }^{e}-X V I I^{e}$ siècles), Thèse, Université Paris IV.

WUNDERLI, P., 1980, «Le système des démonstratifs en moyen français », Romania, 101, p. 1-34. 